\title{
Dense point cloud acquisition with a low-cost Velodyne VLP-16
}

\author{
Jason Bula $^{1}$, Marc-Henri Derron ${ }^{2}$, and Gregoire Mariethoz ${ }^{1}$ \\ ${ }^{1}$ Institute of Earth Surface Dynamics, University of Lausanne, Lausanne, Switzerland \\ ${ }^{2}$ Institute of Earth Sciences, University of Lausanne, Lausanne, Switzerland
}

Correspondence: Jason Bula (jason.bula@unil.ch)

Received: 23 February 2020 - Discussion started: 4 March 2020

Revised: 22 June 2020 - Accepted: 13 August 2020 - Published: 12 October 2020

\begin{abstract}
This study develops a low-cost terrestrial lidar system (TLS) for dense point cloud acquisition. Our system consists of a VLP-16 lidar scanner produced by Velodyne, which we have placed on a motorized rotating platform. This allows us to continuously change the direction and densify the scan. Axis correction is performed in post-processing to obtain accurate scans. The system has been compared indoors with a high-cost system, showing an average absolute difference of $\pm 2.5 \mathrm{~cm}$. Stability tests demonstrated an average distance of $\pm 2 \mathrm{~cm}$ between repeated scans with our system. The system has been tested in abandoned mines with promising results. It has a very low price (approximately USD 4000) and opens the door to measuring risky sectors where instrument loss is high but information valuable.
\end{abstract}

\section{Introduction}

Over these last decades, remote sensing and associated technologies have been developed and used to greatly improve environmental modelling. In particular, light detection and ranging (hereafter lidar) has been proposed as a tool in geomatics to address such environmental modelling. Lidar technology is based on the time of flight (ToF, i.e. the time required by the light emitted by the laser to be reflected and captured again by the system) to measure distances. Lidar is useful for solving many problems. It is therefore widely used in geosciences, in particular for the management and the monitoring of environmental risks such as landslides, rock falls or cavity collapse (Lim et al., 2005; Teza et al., 2007; Jaboyedoff et al., 2011; Brideau et al., 2012; Royán et al., 2014; Michoud et al., 2015). The reliability of these measuring instruments is well established, but the technology is typ- ically very expensive, which limits the potential applications of such systems.

New lidar-based obstacle avoidance technologies have been under development since the advent of autonomous cars. These mass-produced sensors are cheap but were not initially designed to produce dense point clouds and therefore have reduced ranges and resolutions. These low-cost systems have led to the development of new scanner systems that can be applied for mapping, especially for mobile terrestrial SLAM-based systems (James and Quinton, 2014; Dewez et al., 2017) or unmanned aerial vehicle (UAV) SLAM-based systems (Laurent et al., 2019; Li et al., 2014) and often require the addition of an inertial station and Global Navigation Satellite System (GNSS).

Instruments used in geodesy such as theodolites or total stations must be calibrated to avoid measurement errors. This principle is also applied to lidar, which is constructed in a similar way. Lidar system calibration is a much studied subject in research. The aim is to determine the parameters that allow systematic errors to be reduced as much as possible. According to Neitzel (2006), three major errors may be present: tilting axis error, collimation error and eccentricity of the line of sight. Some authors describe up to 21 possible calibration parameters (Lichti, 2007). There are different strategies for calibrating a lidar system. Research proposes a self-calibration based on mathematical models and by making geometric primitives reference planes (Glennie and Lichti, 2010; Lerma and Garcia-San-Miguel, 2014) or reference points (Neizel, 2006; Kersten et al., 2005). Other authors present calibration methods based on the use of a camera (Amiri Parian and Grün, 2005; Lichti et al., 2007).

Our study is based on the use of a low-cost lidar system, which is the VLP-16 of Velodyne, to elaborate on a low-cost terrestrial lidar system (TLS). This scanner, currently sold 
Table 1. Velodyne VLP-16.

\begin{tabular}{ll}
\hline Channel & 16 \\
Wavelength & $903 \mathrm{~nm}$ \\
Ranging accuracy & $\pm 3 \mathrm{~cm}$ (typical) \\
Measurement range & Up to $100 \mathrm{~m}$ \\
Single return data points & 300000 points s$^{-1}$ \\
Maximum number of returns & 2 \\
Field of view (vertical) & $30^{\circ}$ \\
Vertical angular resolution & $2.0^{\circ}$ \\
Field of view (horizontal) & $360^{\circ}$ \\
Horizontal angular resolution & $0.1-0.4^{\circ}$ \\
Laser rotation & $10 \mathrm{~Hz}$ \\
Weight & $830 \mathrm{~g}$ \\
Dimension & $\varnothing 103 \mathrm{~mm}, h 72 \mathrm{~mm}$ \\
Retail price & $\mathrm{USD} 4000$ \\
\hline
\end{tabular}

for USD 4000, has 16 parallel scan lines in a vertical field of view of $\pm 15^{\circ}$ and a $360^{\circ}$ horizontal scan plane (Fig. 2c). Our idea was based on the addition of a rotating plate (which is a principle similar to that of many lidar systems) to produce a dense point cloud. Such a system particularly targets applications in rough field conditions, such as caves that are difficult to access. In such places there is the likelihood of damage to the equipment due to shocks, water or dirt, which prevents the use of high-cost equipment. This type of system could also facilitate risk management in mines for the development of cave collapse risk maps, for example. The advantage is that the system is inexpensive, making it particularly suitable for permanent laser scanning in hazardous areas as described in Williams et al. (2018). In addition, the power consumption of low-cost lidars is often very small, which is suitable for such environments.

The structure of this paper is as follows: Sect. 2 presents the equipment and the constraints associated with it to produce a low-cost system. Section 3 presents the methodology used to produce high-resolution scans. Section 4 presents the result of our system. Section 5 discusses the results and Sect. 6 presents some conclusions.

\section{Low-cost hardware}

\section{$2.1 \quad$ VLP-16 lidar}

The VLP-16 model has 16 lasers fixed on a rotational head. The main features of the low-cost lidar can be found in Table 1.

Each of the 16 parallel scan lines records up to 1875 points every tenth of a second, which corresponds to an angular horizontal resolution of $0.2^{\circ}$ for a field of view of $360^{\circ}$. Regarding the vertical resolution, the sensor is limited to a field of view of $30^{\circ}$ (Fig. 2c). The 16 scan lines imply a low vertical angular resolution of $2.0^{\circ}$. Each of the 16 lasers in VLP-16 is individually aimed and, thus, each has a unique set of adjust-
Table 2. Syrp Genie.

\begin{tabular}{ll}
\hline Max rotation speed & $0.58 \mathrm{rpm}\left(35 \mathrm{~s}\right.$ for $\left.360^{\circ}\right)$ \\
Min rotation speed & $0.0025 \mathrm{rpm}$ \\
Payload & $4 \mathrm{~kg}$ \\
Hardware Interface & Bluetooth 4.0 \\
Dimensions & $91.5 \mathrm{~mm} \times 91.5 \mathrm{~mm} \times 46 \mathrm{~mm}$ \\
Retail Price & USD 250 \\
Minimum step & $0.005^{\circ}$ \\
\hline
\end{tabular}

ment parameters. The mathematical model of VLP-16 which calculates the $(x, y, z)$ coordinates is given in Glennie and Lichti (2010) as

$$
\begin{aligned}
& {\left[\begin{array}{l}
x \\
y \\
z
\end{array}\right]=} \\
& {\left[\begin{array}{c}
\left(s^{i} \cdot R_{i}+D_{\mathrm{o}}^{i}\right) \cdot \cos \left(\delta_{i}\right) \cdot\left[\sin (\varepsilon) \cdot \cos \left(\beta_{i}\right)-\cos \left(\varepsilon_{i}\right)\right] \\
-H_{\mathrm{o}}^{i} \cdot\left[\cos (\varepsilon) \cdot \cos \left(\beta_{i}\right)+\sin (\varepsilon) \cdot \sin \left(\beta_{i}\right)\right] \\
\left(s^{i} \cdot R_{i}+D_{\mathrm{o}}^{i}\right) \cdot \cos \left(\delta_{i}\right) \cdot\left[\cos (\varepsilon) \cdot \cos \left(\beta_{i}\right)+\sin \left(\varepsilon_{i}\right)\right] \\
+H_{\mathrm{o}}^{i} \cdot\left[\sin (\varepsilon) \cdot \cos \left(\beta_{i}\right)-\cos (\varepsilon) \cdot \sin \left(\beta_{i}\right)\right] \\
\left(s^{i} \cdot R_{i}+D_{\mathrm{o}}^{i}\right) \cdot \sin \left(\delta_{i}\right)+V_{\mathrm{o}}^{i}
\end{array}\right],}
\end{aligned}
$$

where $s^{i}$ is the distance scale factor for laser $i ; D_{\mathrm{o}}^{i}$ is the distance offset for laser $i ; \delta_{i}$ is the vertical rotation correction for laser $i ; \beta_{i}$ is the horizontal rotation correction for laser $i ; H_{\mathrm{o}}^{i}$ is the horizontal offset from scanner frame origin for laser $i$; $V_{\mathrm{o}}^{i}$ is the vertical offset from scanner frame origin for laser $i$; $R_{i}$ is the raw distance measurement from laser $i$; and $\varepsilon$ is the encoder angle measurement.

The first six parameters are used to calibrate the system and can be found in the lidar data sheet. $R_{i}$ and $\varepsilon$ come from data collected during a measurement.

Figure 1a shows a photograph of the scanned scene with the same viewing angle, and Fig. $1 \mathrm{~b}$ shows a typical point cloud produced by the VLP-16 with highlighted vertical and horizontal angular resolutions. The poor vertical resolution limits the use of the VLP-16 for terrestrial scanning applications. For example, the low point density makes it difficult to co-register several scans.

\subsection{Syrp Genie}

For the purpose of having a low-cost design, we select the Syrp Genie Mini (Table 2). This motorized head can rotate $360^{\circ}$ and sustain the weight of the lidar.

\subsection{Conception and assembly of the custom scanning system}

The VLP 16 is mounted on the Syrp Genie Mini, and the entire assembly is set on a photographic tripod and connected to a computer and a power source (Fig. 2a). Importantly, the lidar is placed vertically using an L-shaped piece, such that the vertical (low-resolution) and horizontal (high-resolution) di- 
(a)

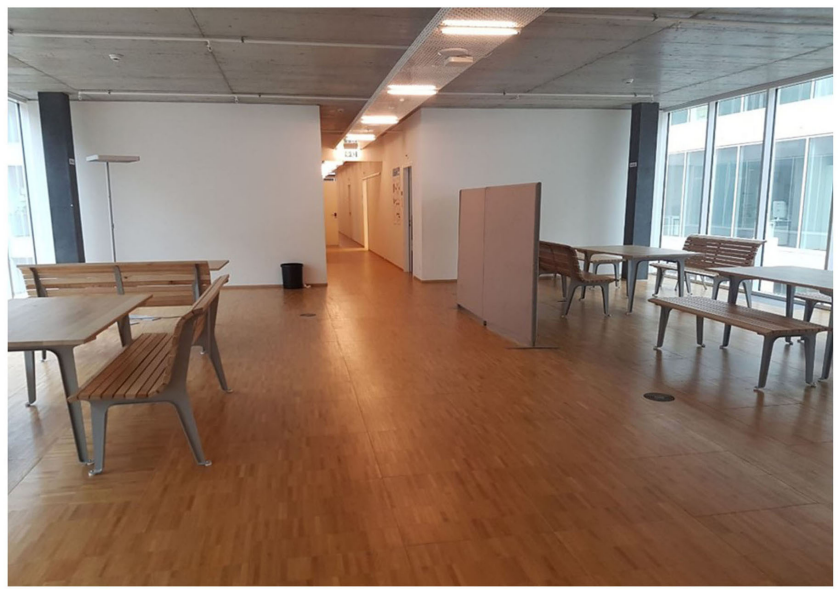

(b)

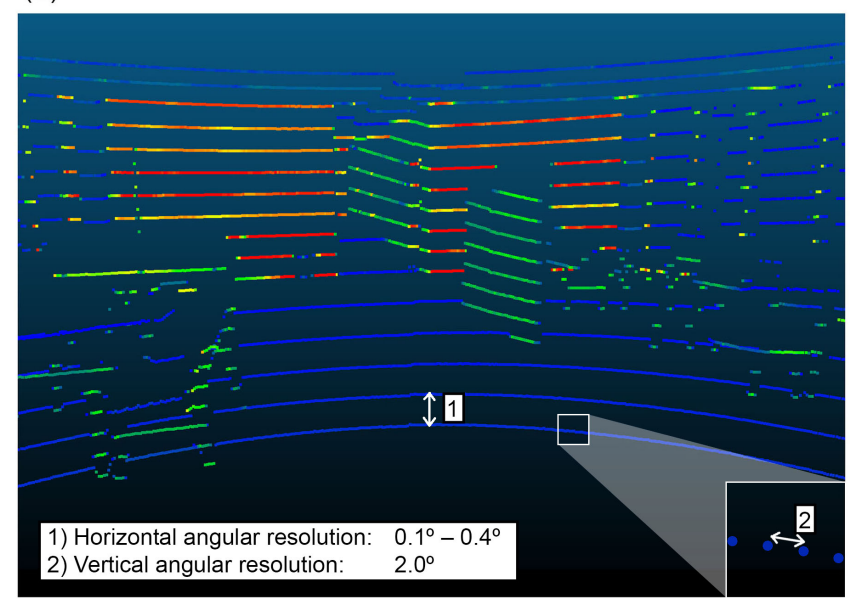

Figure 1. (a) Picture of the scanned scene; (b) a typical scan created with the VLP-16, the colour represents the intensity of the returned signal.

rections are now reversed. It is also important to note that the term "vertical" corresponds to the lidar reference system and not necessarily to the direction of the gravitational field. Our goal is to use the slow rotating motion induced by the Syrp Genie Mini to densify the point cloud across the horizontal direction. It includes a stepper motor with a minimum step of $0.005^{\circ}$ which does not impact the acquisition frequency of the VLP-16. A counterweight is placed on the tripod on the opposite side of the lidar to minimize stresses that can impact the rotation speed and induce an angular distortion.

\section{Methodology}

\subsection{Data acquisition operation}

Acquisition with our system requires a number of postprocessing steps to obtain a scan that correctly represents the scene. For each acquisition, a $360^{\circ}$ rotation is required to re- construct a dense and accurate point cloud; the reason for this will be clarified in Sect. 3.2. In order to ensure that the system does not record the acceleration and deceleration of the motor at the beginning and end of the rotation, the scans are made for a rotation of more than $360^{\circ}$, which allows a better synchronization with the VLP-16. Once the engine starts its rotation, the scan is then started. The post-processing of the data consists of using the set of points (hereafter frame) produced after each rotation of the laser ( 10 rotations per second) and applying to it a transformation in relation to the motor speed. Figure 3 takes the example of a teapot to illustrate densification process, with five steps described below.

1. Frame at time $t=t_{0}$ : only a part of the teapot is scanned, corresponding to the lidar field of view $\left(30^{\circ}\right)$. This first frame is used as a reference to align the others.

2. Scan at time $t=t_{1}$ : a second part of the teapot is scanned.

3. Representation of the scene when both frames are visible simultaneously: it is necessary to apply a transformation to correctly align both frames. This transformation is equal to a rotation on the $y$ axis in clockwise direction by an angle corresponding to the rotation of the motor between $t_{0}$ and $t_{1}$.

4. Reconstructed scene after transformation: both frames are now aligned. Frames are incrementally assembled to construct the entire scene.

5. Visualization of the assemblage of frames acquired between time $t_{0}$ and $t_{f}$.

Assuming a constant geometry of the system, we use a rigid transformation between each frame. This geometrical transformation is characterized by a $4 \times 4$ matrix:

$T=\left[\begin{array}{llll}a & b & c & 0 \\ d & e & f & 0 \\ g & h & i & 0 \\ j & k & l & 1\end{array}\right]$

with $a b c$ is the rotation applied on the $x$ axis; def is the rotation applied on the $y$ axis; $g h i$ is the rotation applied on the $z$ axis; and $j k l$ is the translation applied on $x, y$ and $z$.

In our case, the rotation is applied around the $y$ axis; the transformation matrix that aligns each frame is equal to

$T_{1}=\left[\begin{array}{cccc}(\cos (\beta) & 0 & \sin (\beta) & 0 \\ 0 & 1 & 0 & 0 \\ -\sin (\beta) & 0 & \cos (\beta) & 0 \\ 0 & 0 & 0 & 1\end{array}\right]$,

with $\beta$ the angle of the motor, which depends on the time since the start of the scan and the rotation speed. Once all frames are assembled, the entire point cloud can be visualized. 


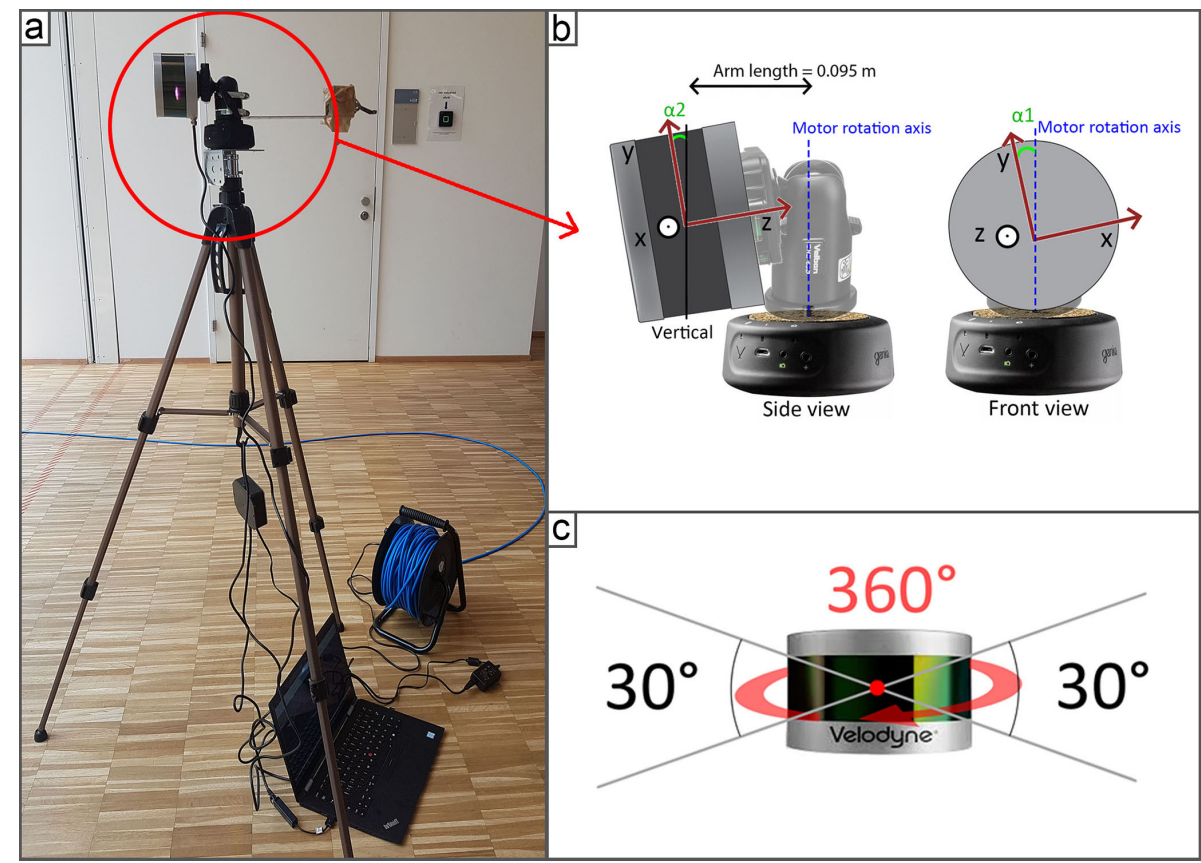

Figure 2. (a) Terrestrial lidar system (TLS); (b) two adjustments angles: collimation axis $\left(\alpha_{1}\right)$ and tilting axis $\left(\alpha_{2}\right)$ between the system and the rotation axis; (c) field of view of the VLP-16.
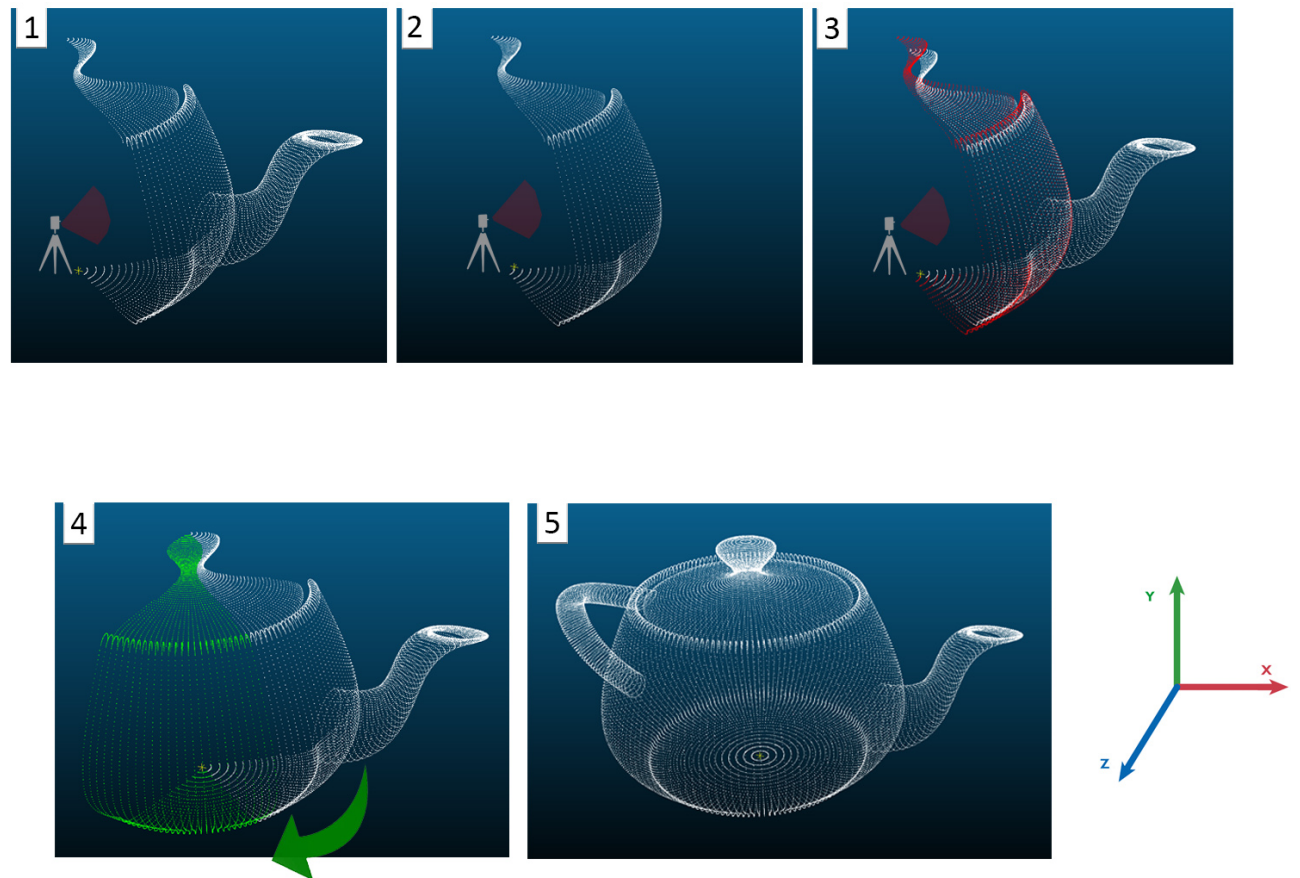

Figure 3. Steps to align the final. This is a synthetic example assuming that the lidar is located in the centre of the teapot point cloud.

As the VLP-16 Puck has the particularity of being able to scan continuously and at $360^{\circ}$, two sets of symmetrical point clouds (transit theodolite design) representing, respectively, the points with positive and negative coordinates on the $x$ axis of the lidar reference frame (see Fig. 5a) are cre- ated, which are theoretically superposed. This observation is a crucial point of the study as it allows the adjustment of the system in order to maximize this superposition (the adjustment procedure is described in Sect. 3.2). 


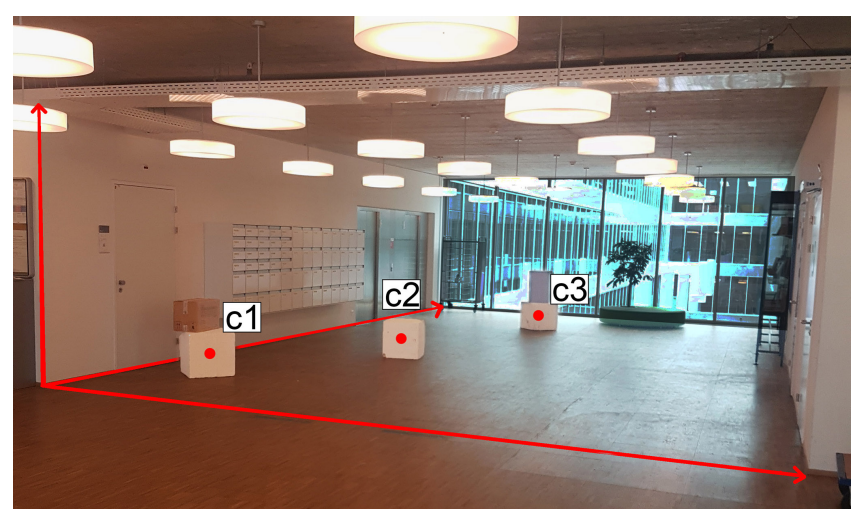

Figure 4. Picture of the scanned scene for lidar and distance comparison. The letters $\mathrm{c} 1, \mathrm{c} 2$ and $\mathrm{c} 3$ represent cubes of $0.5 \mathrm{~m} \times 0.5 \mathrm{~m} \times$ $0.5 \mathrm{~m}$. The red arrows are the length, width and height of the ceiling.

\subsection{Post-processing and data adjustment}

Since our system is custom-assembled, there is little control on exact mounting angles, which therefore require adjustments. Thus far, we have supposed that the system is turning around a fixed point corresponding to its optical centre. In fact, given that the lidar is positioned on a ball head and an L-shaped piece, it is shifted from the rotation axis. This distance was measured using an electronic caliper and is equal to $0.095 \mathrm{~m}$; for each frame a translation on the $z$ axis was applied. The affine transformation is a matrix presented as follows:

$T_{2}=\left[\begin{array}{cccc}1 & 0 & 0 & 0 \\ 0 & 1 & 0 & 0 \\ 0 & 0 & 1 & 0 \\ 0 & 0 & 0.095 & 1\end{array}\right]$.

Another important consideration is the correction to minimize systematic errors. Figure $2 b$ shows two major adjustment parameters described in Neitzel (2006): $\alpha_{1}$ for the collimation axis adjustment and $\alpha_{2}$ for the tilting axis adjustment. The manual adjustment of these two systems involves an offset that greatly influences the point cloud geometry if uncorrected. As these offsets cannot be measured manually, an automatic adjustment is performed in post-processing. To this end, $\alpha_{1}$ and $\alpha_{2}$ are estimated through an optimization procedure that minimizes two functions:

1. During the rotation of the motor, the entire scene is recreated for each of the 16 scan lines. These identical scans are then put back together to form a dense point cloud. The overlap of the scans is influenced by changing the angle $\alpha_{1}$. Thus, the first function to optimize corresponds to minimizing the average distance between the 16 full scenes that are superimposed.

2. The second function determines the angle $\alpha_{2}$ based on the observation that both sets of symmetrical point (a)

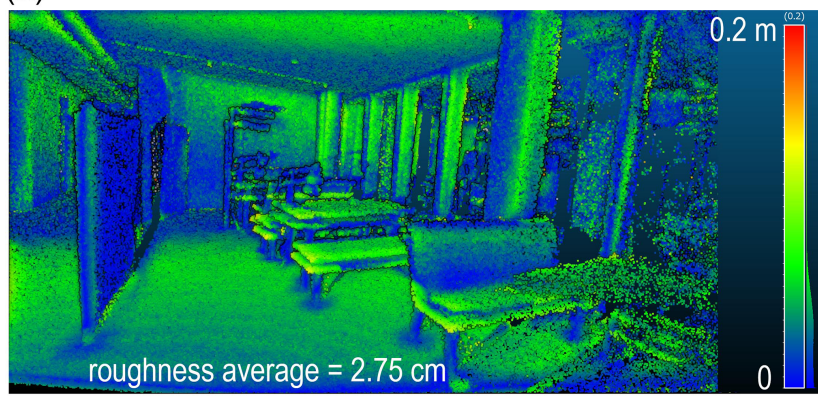

(b)

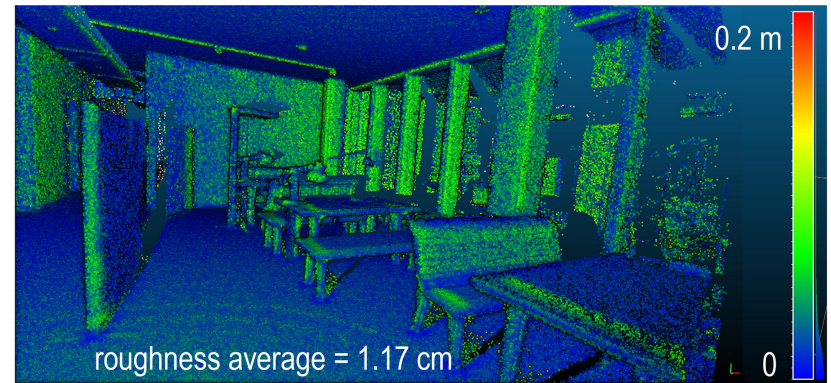

(c)

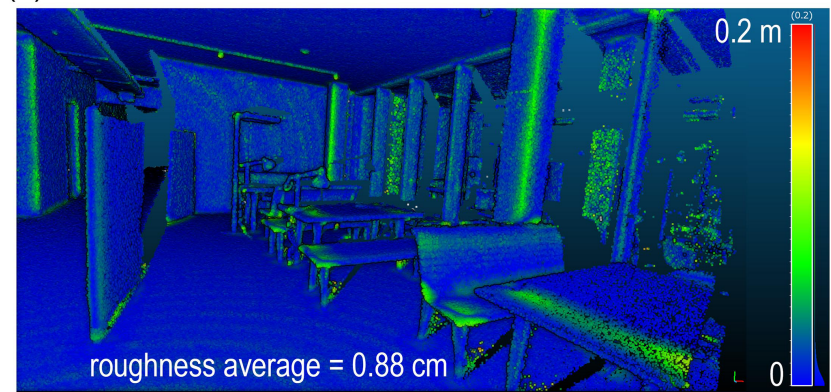

(d)

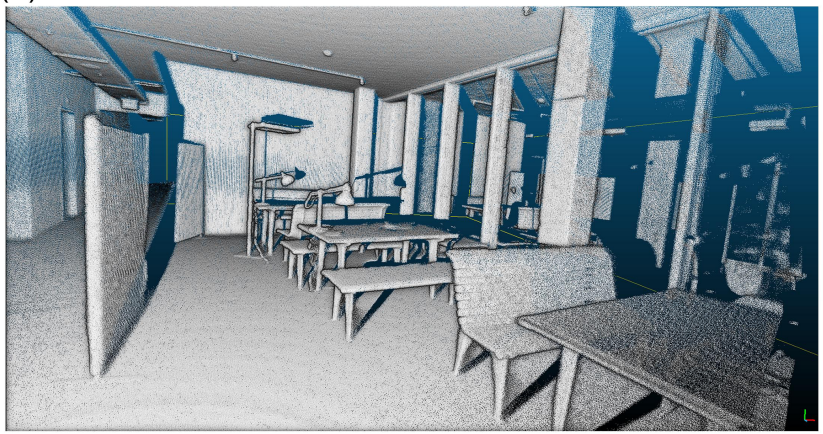

Figure 5. Point cloud roughness for adjustment steps: (a) scan without adjustments; (b) scan after adjustments of $\alpha_{1}$ and $\alpha_{2}$; (c) final scan; (d) final scan (EDL filter).

clouds produced during the rotation must be exactly superposed. The variation in the angle $\alpha_{2}$ creates a doming effect that tends to increase the average distance between both symmetrical point clouds (Fig. 5a). $\alpha_{2}$ is determined by minimizing this distance. 
Optimization is carried out with the Nelder-Mead method, which is based on minimizing a continuous function by evaluating points along a pattern having the shape of a simplex of dimensions equivalent to the number of parameters (Lagarias et al., 1998). The advantage of this approach is that it is derivative-free and is often appropriate when the function to minimize is discontinuous.

The resolution of the densified scan is higher near the lidar scanner and decreases away from the sensor. Because the algorithms for measuring the distance between two sets of point clouds require a lot of computational resources and must be repeated at each iteration of the Nelder-Mead optimization, the scans are downsampled using a grid average method to a uniform resolution. In addition, the optimization is carried out only for points within the distance range in the best accuracy range of 3 to $7 \mathrm{~m}$ (Glennie et al., 2016).

\subsection{Effect of the adjustments and performance of the system}

Visually, a wrong adjustment of $\alpha_{1}$ results in blur around the scanned scene. A wrong adjustment of the $\alpha_{2}$ angle results in a doming effect that increases away from the centre. To illustrate this, several scans were carried out in a building of the University of Lausanne. A corridor of $23 \mathrm{~m} \times 1.5 \mathrm{~m}$ was scanned and a plane was fitted on the floor surface, which is supposed to be horizontal. This plane is based on a distance interval to the lidar equivalent to the best accuracy range, i.e. between 3 and $7 \mathrm{~m}$ depending on the lidar performance tests (Glennie et al., 2016). This avoids the influence of points too close or too far away, which can distort the theoretical equation of the plane. In addition, the points selected for fitting the plane come from an adequate sub-sampling of the initial scan in order to standardize the density of points over the distance interval. Then, the distance of all points to this theoretical plane is evaluated, which gives an indication of the distribution of errors. Evaluation of the error as a function of the scanning distance was also measured.

Finally, reproducibility tests were performed to evaluate the stability of the system. To this end, the same scene was scanned eight times in a row. The $\alpha_{1}$ and $\alpha_{2}$ parameters were then estimated separately. As the system is not transported or disassembled between measurements, the aim of this test was to evaluate the stability of these parameters. The average distance between each of the eight scans is also evaluated using a multiscale model-to-model cloud comparison (M3C2 plugin in CloudCompare with its default settings) which estimates local distance between two point clouds from normal surface direction. It allows us to compute signed (and robust) distances directly between two point clouds.

\subsection{Testing the system in different environments}

The system has been tested in various environments. For all scans performed, the Syrp Genie Mini was configured to ro- tate continuously $360^{\circ}$ in $6 \mathrm{~min}$. These parameters allowed the acquisition of high point resolution to maximize the information collected while maintaining a reasonable scan time. With this setting, about $10 \times 10^{6}$ points per scan are collected. The first tests were carried out in a building of the University of Lausanne, which is characterized by vast surfaces and volumes. Then, the system was then used in a confined environment with no available GNSS signal: the Baulmes mines, a limestone mine abandoned at the end of the Second World War.

In these environments, several scans were assembled using the iterative closest point (ICP) alignment algorithm (Besl and McKay in 1992). This is the most popular alignment approach method for point clouds, which searches for nearest neighbours to minimize the distance between two point clouds.

\subsection{Comparison with a high-cost system and field measurements}

Our low-cost terrestrial lidar system was then compared to two other systems that fall into the high-cost category. These lidar systems are the Ilris 3D Optec (terrestrial laser scanner) and the GeoSLAM ZEB-REVO (mobile laser scanner with IMU tracking) (Table 3). The tests were carried out indoors and included one measurement for each system. First, Fig. 4 displays a picture of the scanned scene. As shown, objects were placed on the scene so that validation measurements could be taken with a tape measure in addition to the width and length of the corridor and the height of the ceiling. These distances were then measured for the scans coming from all three terrestrial lidar systems and compared to the measurements taken by hand. The different scans were also superimposed with an ICP algorithm to evaluate the average distance between the point clouds.

\section{Results}

All point clouds are visualized in the CloudCompare software. An EDL (Eye Dome Lighting) shading filter allowing the creation of real-time shading has been applied for better visualization (CloudCompare, 2019).

\subsection{Effects of adjustments}

Figure 5 shows the result of a scan that was carried out indoors in a work area of the University of Lausanne. Figure 5a shows the scene after the alignment of the different frames produced by the VLP-16 during the scan. A kind of blur caused by the splitting of the scene is observed. No processing has yet been applied, so the parameters $\alpha_{1}$ and $\alpha_{2}$ are set to 0 . The colour scale represents the roughness of the point cloud (for a radius of $0.2 \mathrm{~m}$ ). Figure $5 \mathrm{~b}$ shows the scene after adjusting the $\alpha_{1}$ and $\alpha_{2}$ parameters. The average roughness is equal to $1.17 \mathrm{~cm}$. Figure $5 \mathrm{c}$ shows the scene where 
Table 3. Main characteristics of lidar used.

\begin{tabular}{lrr}
\hline Parameter & Ilris-3D (TLS) & GeoSLAM ZEB-REVO (MLS, mobile lidar scanning) \\
\hline Range & $1200 \mathrm{~m}$ & $30 \mathrm{~m}$ \\
Field of view & $40^{\circ} \times 40^{\circ}$ & $270^{\circ} \times 360^{\circ}$ \\
Ranging accuracy & $7 \mathrm{~mm}^{\circ} 100 \mathrm{~m}$ & $1-3 \mathrm{~cm}$ \\
Laser wavelength & $1535 \mathrm{~nm}$ & $905 \mathrm{~nm}$ \\
Laser acquisition rate & $2500-3500$ points s $^{-1}$ & 43200 points s $^{-1}$ \\
\hline
\end{tabular}

only the scanned points corresponding to the positive coordinates on the $x$ axis are displayed (i.e., $50 \%$ of the data is discarded). In addition, a sub-sampling at $0.005 \mathrm{~m}$ is applied using a grid average method and a statistical outlier removal is performed in CloudCompare. The average roughness is equal to $0.88 \mathrm{~cm}$. Figure $5 \mathrm{~d}$ is a copy of Fig. $5 \mathrm{c}$ with an EDL filter.

Figure 6 shows the error corresponding to the distance of the points from the theoretical plane and the error histogram for the three adjustments steps.

\subsection{Overview of the densification quality}

Figure 7a provides an overview of a scan performed indoors after adjustments. A photograph of the scanned scene with the same viewing angle is shown in Fig. 7b. Note the improvement compared to Fig. 1, where the VLP-16 was used alone.

Figure $8 \mathrm{a}$ and $\mathrm{b}$ show two previews of scans performed at the Baulmes mines. Figure $8 \mathrm{c}$ shows the result of the registration of four point clouds in the Baulmes mines. The points corresponding to each of the acquisitions are represented in a different colour to highlight the registration. It should be noted that the clouds have not been cleaned to removed outliers, so we can see that the sensor has scanned itself. The results are characterized by a spacing set at $0.005 \mathrm{~m}$ and are visually realistic.

\subsection{Data analysis and validation}

During the visualization of the point clouds, we observed the presence of an artefact in all of our measurements. Figure 9 illustrates this artefact, which is characterized by wavelets near the system that fade away with distance.

Table 4 shows the ability of the system to reproduce the same point cloud by comparing eight consecutive measurements. Each of these measurements is compared to the other seven by measuring the average distance between pairs of scans based on CloudtoCloud comparisons in CloudCompare. Table 5 shows the variation in the parameters $\alpha_{1}$ and $\alpha_{2}$ during this reproducibility test. Table 6 summarizes the different measurements made with the VLP-16, Ilris-3D and GeoSLAM. The data are also compared with manual measurements.

To validate the registration, the comparison of the distances between the points coming from our assembly and those coming from GeoSLAM was carried out using CloudCompare with the option "Cloud to cloud distance". The average distance between both point clouds and the SDs of these distances are shown in Table 7.

\section{Discussion}

\subsection{Data analysis before and after the adjustment of parameters $\alpha_{1}$ and $\alpha_{2}$}

As shown in Fig. 5, adjustment is a fundamental step in producing accurate 3-D modelling of an environment.

The use of the two symmetrical datasets produced during an acquisition using the VLP-16 is a key element in the optimization of the system. However, as shown in Fig. 5b, the roughness calculation tells us that the entire point cloud could still be smoother. For this reason, we decided to keep only half of the points (Fig. 5c and d) in order to obtain a sharper representation of the scene. This choice will be justified after the following analysis.

The error depends on the distance to the lidar. Before adjustment, the distance to the theoretical plane varies from about $\pm 4 \mathrm{~cm}(\mu \pm 1 \sigma)$ for the closest points to the lidar to $59 \mathrm{~cm}(\mu \pm 1 \sigma)$ for a scan distance of $23 \mathrm{~m}$ (Fig. 6a). After adjustment of parameters $\alpha_{1}$ and $\alpha_{2}$, the entire point cloud approaches the theoretical surface, as shown in Fig. 6b. However the evolution of the error as a function of distance is still not constant and tends to increase linearly with the scan distance. The minimum error is logically in the point range where the theoretical plane is situated. The bimodal error histogram shown in Fig. $6 \mathrm{~b}$ and centred at $\pm 0.5 \mathrm{~cm}$ shows that the superposition between the two halves of the scan is still not entirely accurate despite the adjustment performed.

The evolution of the error as a function of the scanning distance when keeping only one half of the scan (Fig. 6c) shows an accuracy range of $\pm 2.5 \mathrm{~cm}(\mu \pm 1.5 \sigma)$, which remains within the accuracy range given by Velodyne. The post-processing steps and the adjustment of mounting angles has allowed us to drastically reduce errors; however, the adjustment parameters vary greatly between scans, as shown in Tables 5 and 6 . This can be explained by the assembly of our lidar system being relatively unstable. The impact of the adjustment, and particularly the need to repeat the self- 
(a)
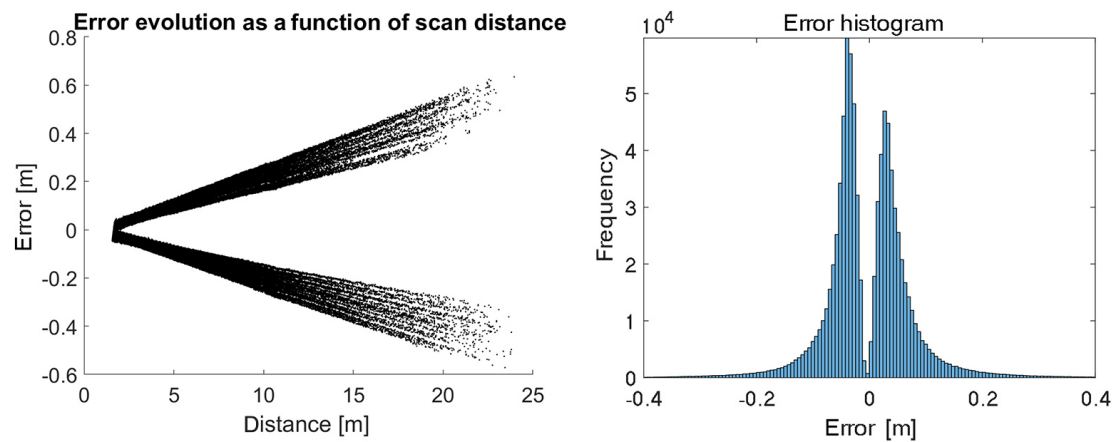

(b)
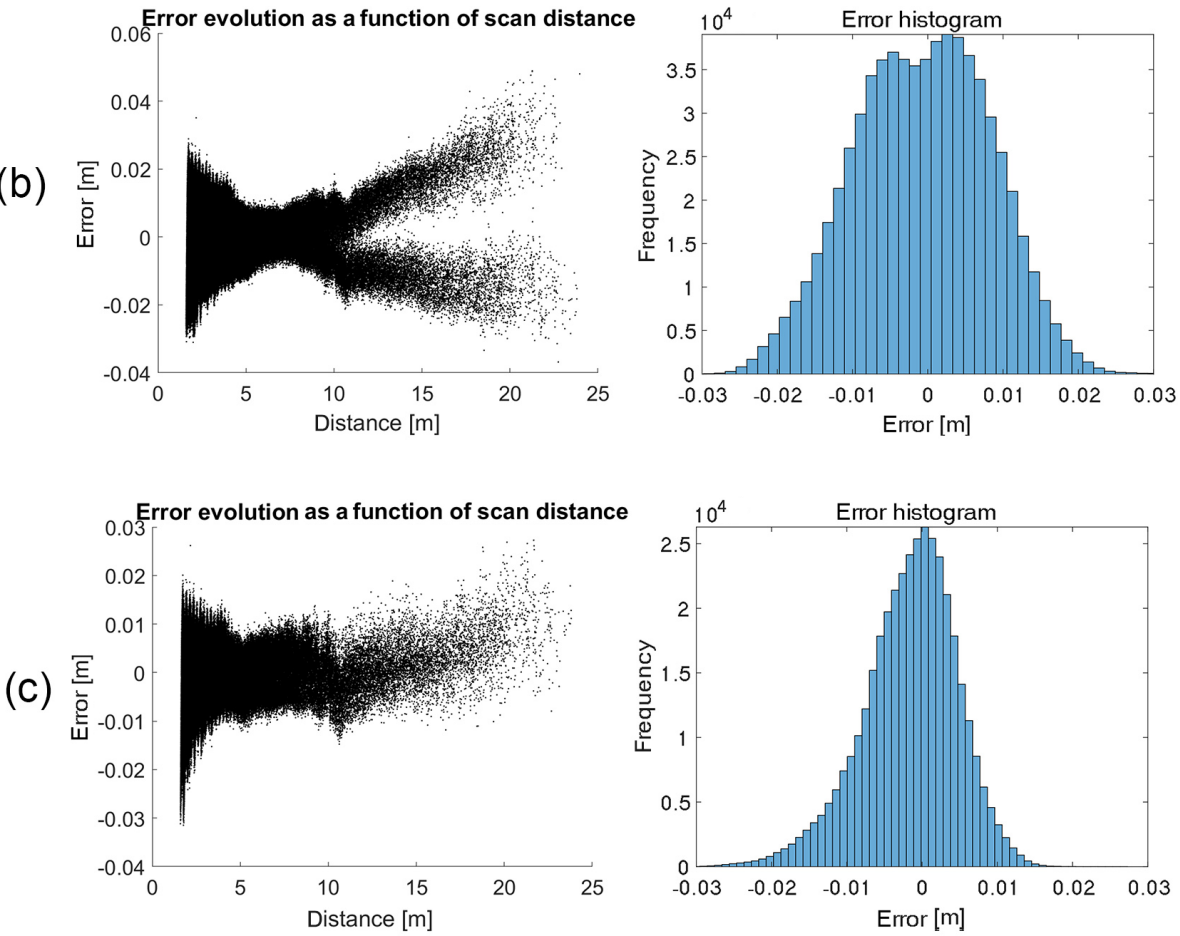

Figure 6. Effects of adjustments in relation to a theoretical plane: (a) error estimation before adjustments; (b) error estimation after adjustments; (c) error estimation after adjustments and post-treatment.

Table 4. Distance between all of the eight scans of the reproducibility test (in $\mathrm{m}$ ).

\begin{tabular}{lcccccccc}
\hline Scan number & 1 & 2 & 3 & 4 & 5 & 6 & 7 & 8 \\
\hline 1 & 0.000 & 0.014 & 0.019 & 0.018 & 0.020 & 0.021 & 0.022 & 0.016 \\
2 & & 0.000 & 0.019 & 0.019 & 0.020 & 0.022 & 0.021 & 0.016 \\
3 & & & 0.000 & 0.013 & 0.015 & 0.016 & 0.016 & 0.019 \\
4 & & & & 0.000 & 0.014 & 0.016 & 0.016 & 0.019 \\
5 & & & & & 0.000 & 0.015 & 0.015 & 0.020 \\
6 & & & & & & 0.000 & 0.015 & 0.022 \\
7 & & & & & & & 0.000 & 0.021 \\
8 & & & & & & & & 0.000 \\
\hline
\end{tabular}


(a)

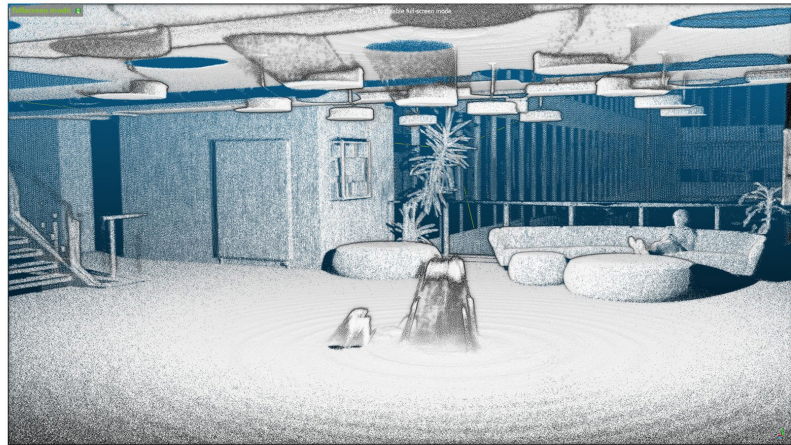

(b)

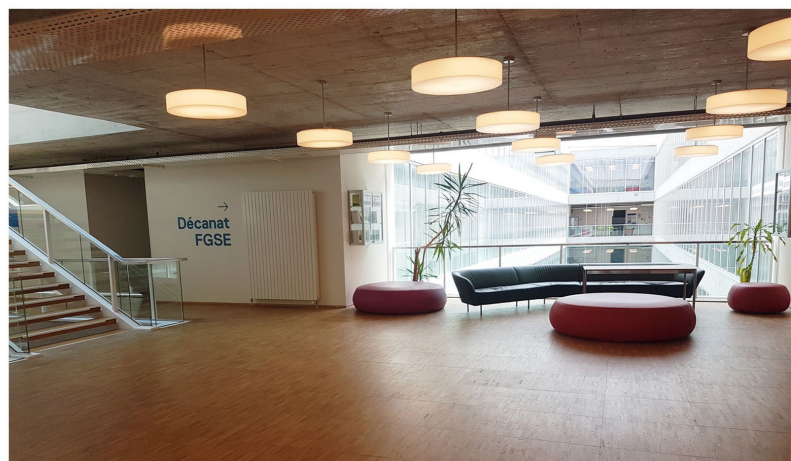

Figure 7. Example of point cloud densification after adjustments of the system: (a) result of an indoor point cloud densification; (b) picture of the scene.

Table 5. Variability of $\alpha_{1}$ and $\alpha_{2}$ parameters during reproducibility test.

\begin{tabular}{lcr}
\hline No. & $\alpha_{1}\left(^{\circ}\right)$ & $\alpha_{2}\left(^{\circ}\right)$ \\
\hline 1 & 0.40 & -0.00 \\
2 & 0.40 & -0.01 \\
3 & 0.09 & -0.03 \\
4 & 0.11 & -0.04 \\
5 & 0.05 & 0.00 \\
6 & 0.05 & 0.00 \\
7 & 0.07 & 0.02 \\
8 & 0.34 & -0.09 \\
\hline
\end{tabular}

adjustment of $\alpha_{1}$ and $\alpha_{2}$, could be alleviated by welding together the different components of the system.

\subsection{Performance and stability of the TLS}

According to the manufacturer's website, the VLP-16 Puck allows data acquisition at a distance of $100 \mathrm{~m}$ for an accuracy of $\pm 3 \mathrm{~cm}$, under optimal acquisition conditions. Various stability tests have been carried out in a metrology laboratory, which indicate an accuracy of $\pm 2 \mathrm{~cm}$ for an acquisition distance of $5 \mathrm{~m}$ to a white and flat target (Glennie et al., 2016).

Reproducibility tests tell us that the system is quite stable when it is not moved or disassembled. The $\alpha_{1}$ adjustment parameter shows a slight variation for scans 1,2 and 8 . This (a)

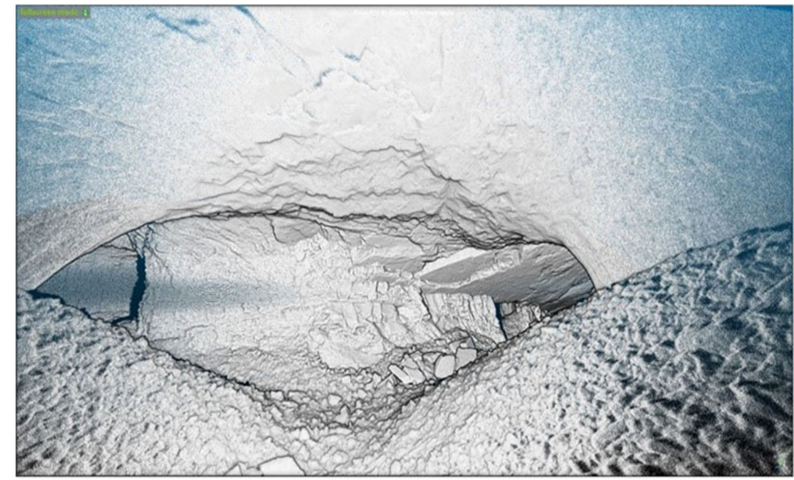

(b)

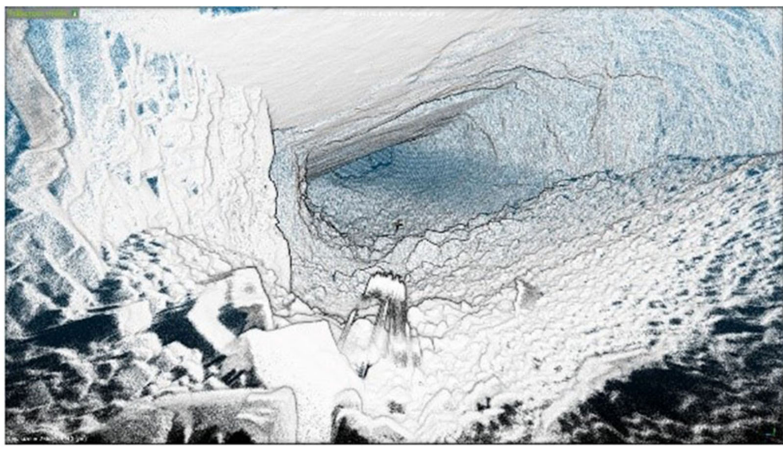

(c)

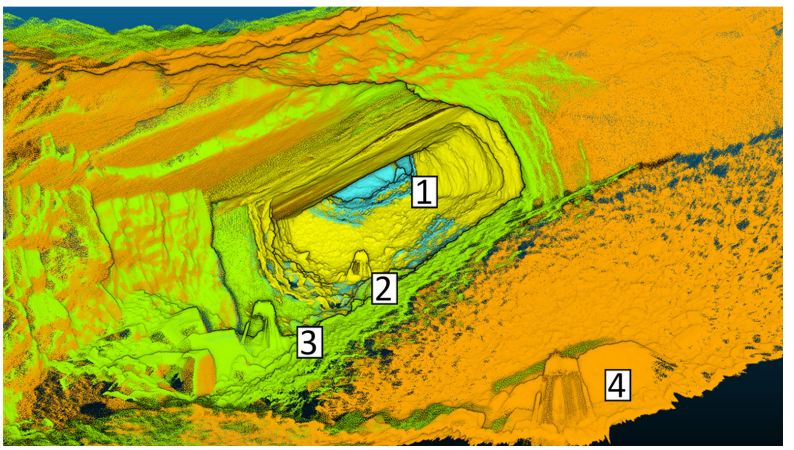

Figure 8. Scanned scenes in Baulmes mines. The height of the gallery is about $3.5 \mathrm{~m}$ : (a) mine example 1; (b) mine example 2; (c) point cloud registration in the mine.

can be explained by the Nelder-Mead algorithm, which finds a local and not a global minimum of the function. The use of this algorithm is nevertheless privileged because of its efficiency in terms of computing time.

The calculation of the average distance between all scans (Table 4) indicates a small variation in the scanned scene. The measured distances are mostly below $2 \mathrm{~cm}$, confirming the tests performed by Glennie et al. (2016) and may be related to noise. We can see a correlation between scan pairs having similar adjustment parameters (scans 1,2,8) and their average distances. 
Table 6. Comparison of field and lidar measurements (see Fig. 4). Vertical line stands for "in comparison with".

\begin{tabular}{lrrrr}
\hline & Field (m) & VLP (m) & Ilris (m) & GeoSLAM (m) \\
\hline Ceiling & 3.005 & 3.001 & 3.003 & 2.964 \\
Length & 11.945 & 11.932 & 11.970 & 11.931 \\
Width & 7.145 & 7.127 & 7.117 & 7.087 \\
\hline Front side of c1 & 0.500 & 0.500 & 0.498 & 0.509 \\
c1-c2 & 2.715 & 2.730 & 2.719 & 2.741 \\
c2-c3 & 4.635 & 4.645 & 4.625 & 4.832 \\
c1-c3 & 7.545 & 7.519 & 7.478 & 7.473 \\
\hline Mean absolute difference | Field (m) & 0 & 0.0123 & 0.0197 & 0.0596 \\
\hline
\end{tabular}

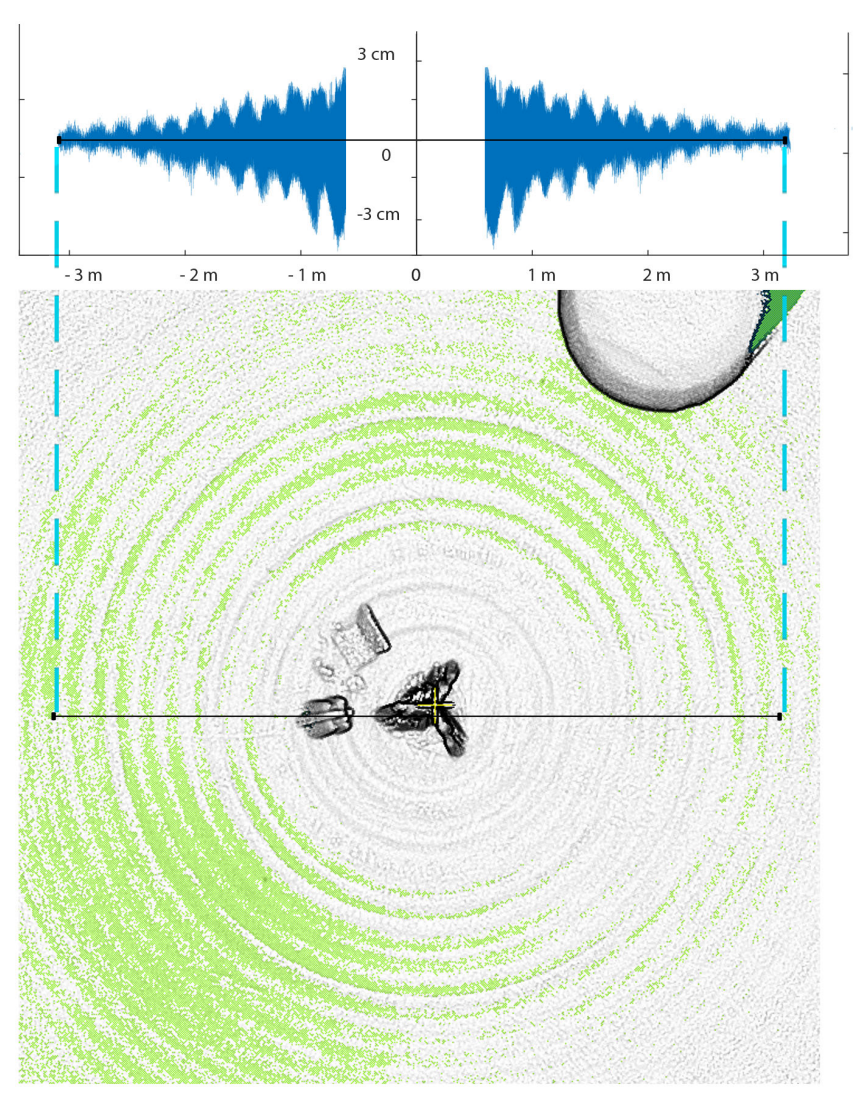

Figure 9. Artefact present near the TLS and its corresponding amplitude.

Table 7. Mean distance between Ilris, GeoSLAM and Velodyne TLS (M3C2 distance).

\begin{tabular}{lr}
\hline & Mean distance (m) \\
\hline Optec Ilris vs. VLP-16 TLS & \pm 0.025 \\
Optec Ilris vs. GeoSLAM & \pm 0.067 \\
VLP-16 TLS vs. GeoSLAM & \pm 0.051 \\
\hline
\end{tabular}

Geosci. Instrum. Method. Data Syst., 9, 385-396, 2020

\subsection{Comparison with high-cost hardware and field measurements}

The measurements taken manually in the field have enabled comparisons with the scans obtained with our system. Table 6 shows that the measurements taken with the VLP-16 TLS are closest to ground truth, with an average of $1.23 \mathrm{~cm}$ compared to $1.97 \mathrm{~cm}$ for the Optec system and $5.96 \mathrm{~cm}$ for GeoSLAM. This result should be treated with caution as it may be specific to our experimental setup. It should also be taken into account that a sub-sampling at $0.5 \mathrm{~cm}$ from the scans was performed using a grid average method.

After alignment using an ICP algorithm, the scans could be compared and are presented in Table 7. Assuming that the Optec system is the most accurate, our system achieves a good performance with an average distance of $\pm 2.5 \mathrm{~cm}$. GeoSLAM obtains a lower level of accuracy with an average of $\pm 6.7 \mathrm{~cm}$.

\subsection{Origin of the short-range artefacts}

Visually, this artefact is easily observed in the results of scans near the tripod, when data were acquired on a flat surface. Figure 9 shows the influence of this artefact on the scan. We notice that the error spreads in the form of regular waves and fades away as it moves away from the lidar. It has a magnitude of $3 \mathrm{~cm}$ closest to the lidar and drops below $1.5 \mathrm{~cm}$ at a distance of $3 \mathrm{~m}$. The wave frequency is about $20 \mathrm{~cm}$. A hypothesis on the origin of this artefact would be related to the length of the arm, which was measured manually. It turns out that errors in the length of the arm have no influence on the occurrence of these artefacts but instead create horizontal deformations. We also tested this by using each laser separately, by using another tripod, and by varying the parameters and changing the motor speed. However, the artefacts remain constant (same distance and amplitude between waves). This indicates that the artefacts seem to be related to the lidar itself. Since the error appears to be regular, it would be conceivable to correct outliers by modifying each point according to the distance from the lidar. 


\section{Conclusions}

This paper presents a low-cost terrestrial lidar system based on the use of a Velodyne VLP-16. Comparisons with highprecision models allow validating the accuracy of the system, which seems promising. As shown in the results, our system requires adjustment for each scan performed. These adjustments are made in post-processing and are possible thanks to the data acquisition geometry of the VLP-16. This could be avoided if the system components were welded together. However, we wanted to keep the possibility of separating these elements in order to use the lidar for other projects, for example. The use of the lidar system in an underground mine demonstrates the potential applications of such a system, in particular in the field of geosciences.

Code availability. The Velodyne TLS is available at https://doi.org/10.5281/zenodo.4060145 (Bula, 2020a).

Video supplement. The following videos represent the result and assembly of several scans performed in the field. The animations were performed on Cloudcompare.

Baulmes: https://vimeo.com/344063864 (last access: 10 August 2020, Bula, 2020c) Exploration of $150 \mathrm{~m}$ of gallery in the former lime mines in Baulmes (Switzerland). Field acquisition was done in about $2 \mathrm{~h}$ from five scans of $6 \mathrm{~min}$ duration. The scan resolution is millimetric.

Reclère: https://vimeo.com/380239565 (last access: 10 August 2020, Bula, 2020d) Exploration of $350 \mathrm{~m}$ of gallery in the caves of Réclère (Switzerland). The acquisition on the field was done in about $1 \mathrm{~h} 30 \mathrm{~min}$ from seven scans of 6 min duration. The resolution of the scans is $1 \mathrm{~cm}$.

Milandre: https://vimeo.com/380040742 (last access: 10 August 2020, Bula, 2020b) Exploration of $80 \mathrm{~m}$ of gallery in the caves of Milandre (Switzerland). The acquisition in the field was done in about $1 \mathrm{~h}$ from four scans of $6 \mathrm{~min}$ duration. The resolution of the scans is millimetric.

Rolex Learning Center: https://vimeo.com/user52420841 (last access: 10 August 2020, Bula, 2020e) Acquisition of the Rolex Learning Center, a building on the EPFL campus in Lausanne. Measurements made in $1 \mathrm{~h} 30 \mathrm{~min}$ from 18 scans of $1 \mathrm{~min} 30 \mathrm{~min}$. The resolution of the scans is $5 \mathrm{~cm}$.

Author contributions. JB developed the methodology, carried out the field experiments and wrote the paper; MHD provided advice and methodological guidance and contributed to the paper; GM proposed the initial framework, provided supervision and contributed to the paper.

Competing interests. The authors declare that they have no conflict of interest.
Acknowledgements. We thank Stephane Affolter, Pierre-Xavier Meury and Eric Gigandet for access to caves for testing our method.

Review statement. This paper was edited by Anette Eltner and reviewed by two anonymous referees.

\section{References}

Amiri Parian, J. and Grün, A.: Integrated laser scanner and intensity image calibration and accuracy assessment, Int. Arch. Photogramm., 36, 18-23, 2005.

Besl, P. J. and McKay, N. D.: Method for registration of 3-D shapes, P. Soc. Photo-Opt. Ins. 1611, 586-606, 1992.

Bula, J.: jason-bula/velodyne_tls: Dense point cloud Velodyne VLP-16 (Version v1), Zenodo, https://doi.org/10.5281/zenodo.4060145, 2020a.

Bula, J.: Milandre - Scan lidar, Vimeo, available at: https://vimeo. com/380040742, last access: 10 August 2020b.

Bula, J.: Mine de Baulmes VLP-16 velodyne, Vimeo, available at: https://vimeo.com/344063864, last access: 10 August 2020c.

Bula, J.: Scan lidar - Réclère, Vimeo, available at: https://vimeo. com/380239565, last access: 10 August 2020d.

Bula, J.: Rolex Learning Center, Vimeo, https://vimeo.com/ user52420841, last access: 10 August 2020e.

Brideau, M.-A., Sturzenegger, M., Stead, D., Jaboyedoff, M., Lawrence, M., Roberts, N. J., Ward, B. C., Millard, T. H., and Clague, J. J.: Stability analysis of the 2007 Chehalis lake landslide based on long-range terrestrial photogrammetry and airborne LiDAR data, Landslides, 9, 75-91, https://doi.org/10.1007/s10346-011-0286-4, 2012.

Dewez, T. J. B., Yart, S., Thuon, Y., Pannet, P., and Plat, E.: Towards cavity-collapse hazard maps with Zeb-Revo handheld laser scanner point clouds, Photogramm. Rec., 32, 354-376, https://doi.org/10.1111/phor.12223, 2017.

Glennie, C. and Lichti, D. D.: Static Calibration and Analysis of the Velodyne HDL-64E S2 for High Accuracy Mobile Scanning, Remote Sens.-Basel, 2, 1610-1624, https://doi.org/10.3390/rs2061610, 2010.

Glennie, C. L., Kusari, A., and Facchin, A.: Calibration and stability analysis of the VLP-16 laser scanner, Int. Arch. Photogramm. XL-3/W4, 55-60, https://doi.org/10.5194/isprs-archives-XL-3W4-55-2016, 2016.

Jaboyedoff, M., Oppikofer, T., Abellán, A., Derron, M.-H., Loye, A., Metzger, R., and Pedrazzini, A.: Use of LIDAR in landslide investigations: a review, Nat. Hazards, 61, 5-28, https://doi.org/10.1007/s11069-010-9634-2, 2012.

James, M. R. and Quinton, J. N.: Ultra-rapid topographic surveying for complex environments: the hand-held mobile laser scanner (HMLS): Ultra-Rapid Topographic Surveying: The Hmls, Earth Surf. Proc. Land., 39, 138-142, https://doi.org/10.1002/esp.3489, 2014.

Kersten, T., Sternberg, H., and Mechelke, K.: Investigations into the accuracy behaviour of the terrestrial laser scanning system Mensi GS100, P. Soc. Photo-Opt. Ins., VII.1, 122-131, 2005.

Lagarias, J. C., Reeds, J. A., Wright, M. H., and Wright, P. E.: Convergence Properties of the Nelder-Mead Simplex 
Method in Low Dimensions, SIAM J. Optimiz., 9, 112-147, https://doi.org/10.1137/S1052623496303470, 1998.

Laurent, A., Moret, P., Fabre, J. M., Calastrenc, C., and Poirier, N.: La cartographie multi-scalaire d'un habitat sur un site accidenté: la Silla del Papa (Espagne), NUMEAR, 3, https://doi.org/10.21494/ISTE.OP.2019.0352, 2019.

Lerma, J. L. and García-San-Miguel, D.: Self-calibration of terrestrial laser scanners: selection of the best geometric additional parameters, ISPRS Ann. Photogramm. Remote Sens. Spatial Inf. Sci., II-5, 219-226, https://doi.org/10.5194/isprsannals-II-5-2192014, 2014.

Li, R., Liu, J., Zhang, L., and Hang, Y.: LIDAR/MEMS IMU integrated navigation (SLAM) method for a small UAV in indoor environments, in: 2014 DGON Inertial Sensors and Systems (ISS), IEEE, Karlsruhe, Germany, 1-15 September 2014.

Lichti, D. D.: Error modelling, calibration and analysis of an AMCW terrestrial laser scanner system, ISPRS J. Photogramm., 61, 307-324, https://doi.org/10.1016/j.isprsjprs.2006.10.004, 2007.

Lichti, D., Brustle, S., and Franke, J.: Self-calibration and analysis of the Surphaser 25HS 3D scanner, in: Proceedings of the Strategic Integration of Surveying Services, FIGWorkingWeek, Hong Kong SAR, China, 13-17 May 2007.

Lim, M., Petley, D. N., Rosser, N. J., Allison, R. J., Long, A. J., and Pybus, D.: Combined Digital Photogrammetry and Time-of-Flight Laser Scanning for Monitoring Cliff Evolution, Photogramm. Rec., 20, 109-129, https://doi.org/10.1111/j.14779730.2005.00315.x, 2005.
Michoud, C., Carrea, D., Costa, S., Derron, M.-H., Jaboyedoff, M., Delacourt, C., Maquaire, O., Letortu, P., and Davidson, R.: Landslide detection and monitoring capability of boat-based mobile laser scanning along Dieppe coastal cliffs, Normandy, Landslides, 12, 403-418, https://doi.org/10.1007/s10346-014-0542-5, 2015.

Neitzel, F.: Investigation of Axes Errors of Terrestrial Laser Scanners, Fifth International Symposium Turkish-German Joint Geodetic Days, Berlin, Germany, 29-31 March 2006.

Northend, C. A., Honey, R. C., and Evans, W. E.: Laser Radar (Lidar) for Meteorological Observations, Rev. Sci. Instrum., 37, 393-400, https://doi.org/10.1063/1.1720199, 1966.

Royán, M. J., Abellán, A., Jaboyedoff, M., Vilaplana, J. M., and Calvet, J.: Spatio-temporal analysis of rockfall pre-failure deformation using Terrestrial LiDAR, Landslides, 11, 697-709, https://doi.org/10.1007/s10346-013-0442-0, 2014.

Teza, G., Galgaro, A., Zaltron, N., and Genevois, R.: Terrestrial laser scanner to detect landslide displacement fields: a new approach, Int. J. Remote Sens., 28, 3425-3446, https://doi.org/10.1080/01431160601024234, 2007.

Williams, J. G., Rosser, N. J., Hardy, R. J., Brain, M. J., and Afana, A. A.: Optimising 4-D surface change detection: an approach for capturing rockfall magnitude-frequency, Earth Surf. Dynam., 6, 101-119, https://doi.org/10.5194/esurf-6-101-2018, 2018. 